\title{
Synthesis of ZSM-22 in Static and Dynamic System Using Seeds
}

\author{
Lenivaldo V. de Sousa Júnior, Antonio O. S. Silva, Bruno J. B. Silva, Soraya L. Alencar \\ Chemical Engineering Department, Federal University of Alagoas, Laboratory Synthesis Catalysts (LSCat), \\ Maceió, Brazil \\ Email: lenivaldovalerio@hotmail.com
}

Received 27 November 2013; revised 27 December 2014; accepted 5 January 2014

Copyright (C) 2014 by authors and Scientific Research Publishing Inc.

This work is licensed under the Creative Commons Attribution International License (CC BY). http://creativecommons.org/licenses/by/4.0/

(c) (i) Open Access

\begin{abstract}
ZSM-22 was synthesized using various sources of silica, organic template 1,6-diaminohexane, under hydrothermal conditions, with and without agitation during crystallization. Subsequently, the crystallized material was used as seeds to accelerate the crystallization process. Characterization of the ZSM-22 samples was performed by XRD, ATG/DTG and FT-IR. It was found that it is possible to synthesize ZSM-22 employing colloidal silica and pyrolytic silica as silicon sources only if the system is stirred during crystallization. The crystallization time for these systems was 13 hours, longer times of crystallization do not significantly increase the crystallinity of the sample. The addition of seeds significantly accelerates the crystallization of ZSM-22, reducing the crystallization time to only 7 hours, with stirring and with systems employing colloidal silica.
\end{abstract}

\section{Keywords}

ZSM-22; Seeds; Zeolite; 1,6-Diaminohexane

\section{Introduction}

ZSM-22 belongs to the group of medium-pore zeolite (mordenite family) which includes ZSM-5, ZSM-11, ZSM-35. It is rich in five-membered rings with wavy channels surrounded by ten-membered rings. The opening of channels (free diameter) is $0.45 \times 0.55 \mathrm{~nm}$ running through the structure in a single crystallographic direction, having no intersections of the channels. The ten members channels of ZSM-22 are smaller than those found in ZSM-5, ZSM-11 and ZSM-35, however the determination of the structure of ZSM-22 indicated that its topology is similar to that of zeolite Theta- 1 (TON structure type) is also similar to that found in the materials: $\mathrm{Nu}-10$, ISI-1, KZ-2.

ZSM-22 in acid form is used in reactions such as: selective formation of p-dialquilbenzenes, enriched in 
p-xylene C8 aromatics, alkylation of toluene with methanol selective for p-xylene and isomerization of 1-butene are particularly desirable in the petroleum industry. The acidic properties and selectivity of ZSM-22 have been addressed by many researchers in their studies [1]-[4].

Seed-assisted crystallization is a very useful method and has been used since the synthesis of the first zeolites in the mid-1960s, where the main function is to provide the system with the area over which the products are to be developed, avoiding the need for generation of this surface by the system itself, through primary nucleation. Its main effect is a reduction of the synthesis time, due to increased crystallization rate, resulting in materials with low impurity content [5] [6].

This paper studied the synthesis of ZSM-22, using 1,6-diaminohexane as the organic template, various sources of silica, with the $\mathrm{SiO}_{2} / \mathrm{Al}_{2} \mathrm{O}_{3}$ ratio constant. Thereafter, the zeolite crystals were used as seed for further syntheses.

The challenge is to synthesize a copy of pure ZSM-22, since ZSM-5 and cristobalite impurities are difficult to avoid [7]. Different sources of silica are studied because their dissolution and reaction temperature determine the rate of crystallization of zeolite. Colloidal silica reacts rapidly to temperatures below $170^{\circ} \mathrm{C}$ while the pyrolytic silica reacts readily at $170^{\circ} \mathrm{C}$ [8].

\section{Experimental}

\subsection{Synthesis of Zeolite ZSM-22 with Different Sources of Silica}

For synthesis the following reagents were used: 1,6-diaminohexane as organic template (Sigma-Aldrich, 98\%), potassium hydroxide (Sigma-Aldrich, 85\%), colloidal silica (Ludox AS40, Sigma-Aldrich), pyrolytic (Fumed) silica (0.014 $\mu$, sigma-aldrich), silica gel (Diasil 200, Diatom), cogel from the precipitation of silica of the commercial sodium silicate (Pernambuco chemical), aluminum sulfate hydrate (Merck) and distilled water.

The gel had the following molar composition: $27 \mathrm{NH}_{2}\left(\mathrm{CH}_{2}\right)_{6} \mathrm{NH}_{2}: 13.5 \mathrm{~K}_{2} \mathrm{O}: \mathrm{Al}_{2} \mathrm{O}_{3}: 90 \mathrm{SiO}_{2}: 3600 \mathrm{H}_{2} \mathrm{O}$, obtained following the methodology:

1) Solution A: Potassium hydroxide is dissolved in $30 \%$ of the total amount of water required for the synthesis;

2) Solution B: 1,6-diaminohexane is solubilized in 30\% of the water required for the synthesis;

3) Solution C: aluminum sulphate is dissolved in $30 \%$ of the water required for the synthesis;

4) Solution D: silica dispersed in the remaining water of synthesis.

The procedure for preparation of the gel consists of: 1) adding solution B to solution A; 2) addition of the solution C; and 3) finally adding the solution D. At each addition step the system was agitated vigorously (with a mechanical stirrer) for $5 \mathrm{~min}$ at $400 \mathrm{rpm}$. The final gel obtained remained in this condition for 30 additional minutes.

For the agitated synthesis, the gel was transferred to a Teflon vessel with a volume of $700 \mathrm{~mL}$. This container was placed inside a stainless steel vessel (with a volume of $1000 \mathrm{~mL}$ ) in a Parr reactor model 4520 . The reaction mixture was stirred at $400 \mathrm{rpm}$ with a double helix impeller (with 6 blades on each rotor) while the system was heated from room temperature to $160^{\circ} \mathrm{C}$ (within two hours).

For the static synthesis, the gel was placed in a Teflon vessel with a volume of $70 \mathrm{~mL}$, and placed inside coated stainless steel autoclaves. Crystallization occurs at a temperature of $160^{\circ} \mathrm{C}$ for periods up to 24 hours.

\subsection{Synthesis of Zeolite ZSM-22 Using Seeds}

The preparation method of the mixture and the synthesis conditions are the same as those described in the previous section. The seeds are added after mixing the solutions A-D, in the amount of $0.71 \mathrm{wt} \%$ of the synthesis gel, and stirred for 15 minutes. Thus, the molar composition of synthesis was: $27 \mathrm{NH}_{2}\left(\mathrm{CH}_{2}\right)_{6} \mathrm{NH}_{2}: 13.5 \mathrm{~K}_{2} \mathrm{O}$ : $\mathrm{Al}_{2} \mathrm{O}_{3}: 90 \mathrm{SiO}_{2}: 3600 \mathrm{H}_{2} \mathrm{O}$ plus $0.71 \mathrm{wt} \%$ seed.

\subsection{Recovery of the Solid Phase after Crystallization}

After crystallization, the autoclave was removed from the oven and cooled to room temperature. The content was transferred to a beaker containing $100 \mathrm{ml}$ of distilled water and the resulting solid was separated from the supernatant liquid by vacuum filtration, washed several times with distilled water (until the $\mathrm{pH}$ of the filtrate reaches the value $\sim 7.0$ ) and dried in an oven at $120^{\circ} \mathrm{C}$ for $12 \mathrm{~h}$. In the case of the agitated synthesis (Parr reac- 
tor), the reactor vessel was cooled by immersion in an ice-water mixture to speed up the washing procedure and the cooling and recovering the solid phase was similar to that employed in the static synthesis.

\subsection{Characterization}

The samples were subjected to X-ray diffraction (XRD) Shimadzu, model XRD-6000. The XRD profile was used to identify the material (type of crystal structure), the calculation of the crystallinity of the material was performed using Equation (1), using the peaks of $2 \theta$ regions of 19.7 to 20.9 and from 23.6 to 25 degrees.

$$
\text { Crist. } \%)=\left(\frac{\sum \text { peak area of the sample }}{\sum \text { peak area of standard sample }}\right)
$$

The verification of presence of contaminating phases was done by comparison with published data in the literature and the diameter of the crystals [9], was done based on the Scherrer Equation (2), where the peak was chosen in the range of 19.7 to 20.9 degrees $2 \theta$.

$$
D_{h k l}=\frac{K \lambda}{\beta \cos (\theta)}
$$

The thermogravimetric analyses (ATG/DTG) were performed in the thermobalance Shimadzu model DTG-60H, with a heating rate $10^{\circ} \mathrm{C} / \mathrm{min}$ in an atmosphere of synthetic air with a flow rate of $100 \mathrm{~mL} / \mathrm{min}$. From these analyses it was possible to quantify the organic template molecules and intracrystalline water present in the solid recovered from the crystallization process.

The absorption spectra in the infrared region (IR) were obtained with a spectrophotometer Fourier transform infrared Shimadzu, Model IRPrestige-21. The spectra were obtained in the range $400-4000 \mathrm{~cm}^{-1}$ by using $\mathrm{KBr}$ as a dispersing agent.

\section{Results and Discussion}

The initial phase of the study consisted of four syntheses varying the silicon source and keeping all other experimental parameters identical. The crystallization was carried out with stirring at $400 \mathrm{rpm}$, at $160^{\circ} \mathrm{C}$ for 24 hours. Figure 1 shows the X-ray diffraction (XRD) of the samples synthesized with colloidal silica and pyrolytic silica. From the comparison with the literature diffraction pattern [10] materials have been identified as zeolite ZSM-22 (TON structure).

The sample synthesized with colloidal silica had a higher crystallinity ( 6\%) when compared with the solid synthesized with pyrolytic silica.

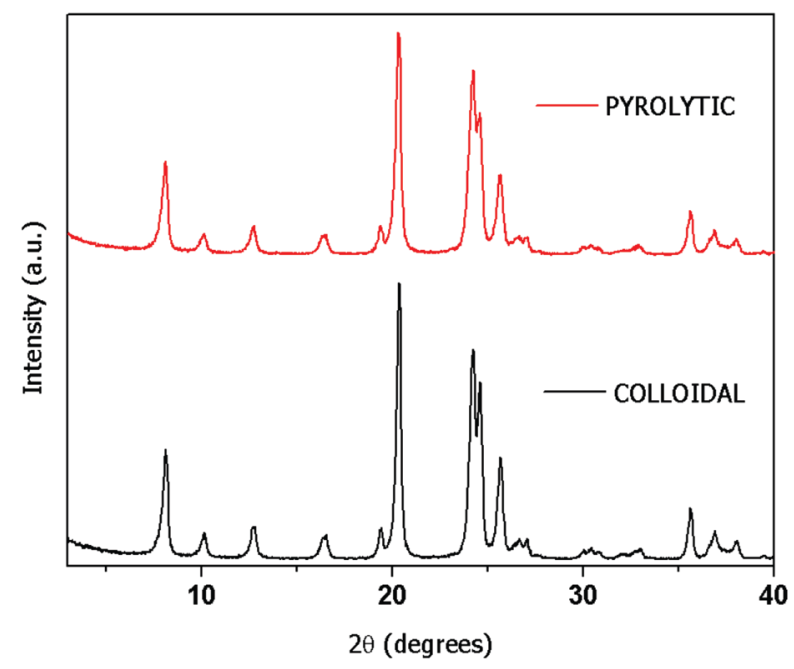

Figure 1. XRD of the samples crystallized under agitation employing colloidal silica and pyrolytic silica. 
The samples with silica gel and cogel (compound solution resulting from the addition of aluminum sulfate + sulfuric acid diluted in a solution of commercial sodium silicate followed by filtration and washing) formed, respectively, the ZSM-5 zeolite and amorphous materials, as shown in Figure 2.

To verify the role of agitation on the crystallization of ZSM-22, a portion of the synthesis gel used in the experiments described above was transferred to a $70 \mathrm{~mL}$ autoclave and subjected to heating at $160^{\circ} \mathrm{C}$ for 24 hours under static conditions. The results of the analysis of X-ray diffraction indicated that for all sources of silica studied there was no formation of crystalline material (only an amorphous solid). A similar result was obtained by Derewinski \& Machowska [11] for synthesis with colloidal silica utilizing the same organic template used in this study.

The sample of ZSM-22 synthesized with colloidal silica with the highest crystallinity was identified, and then grinded and treated by ultrasound for subsequent use as seed material.

The gel composition chosen to assess the role of the seeds was the same as the one used to generate the seed, i.e. a system with colloidal silica and the same amount of organic template. The crystallization conditions were similar to those described previously for static and agitated experiments. Figure 3 shows the XRD of the samples obtained with $24 \mathrm{~h}$ of synthesis.

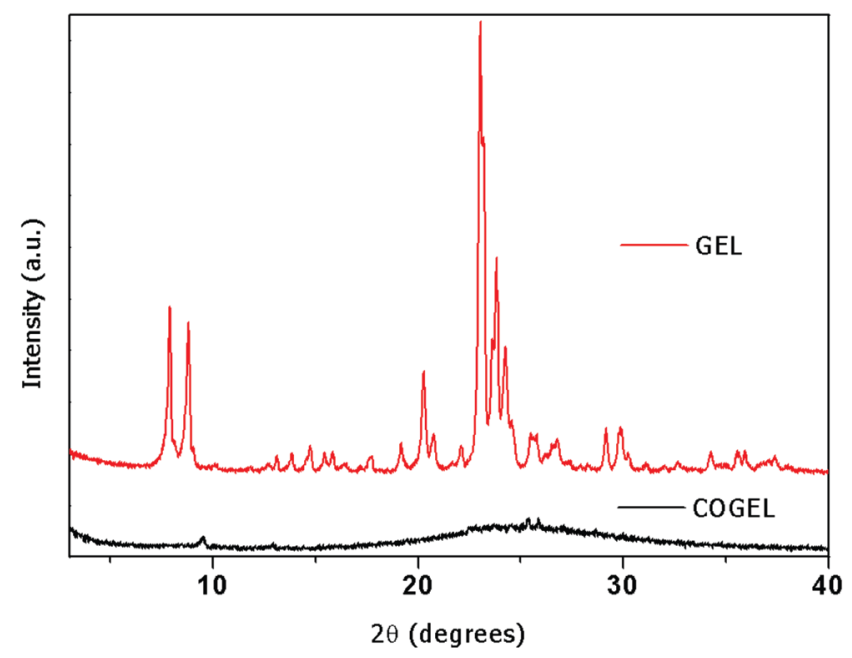

Figure 2. XRD of the samples crystallized with stirring using silica gel and the cogel obtained from sodium silicate.

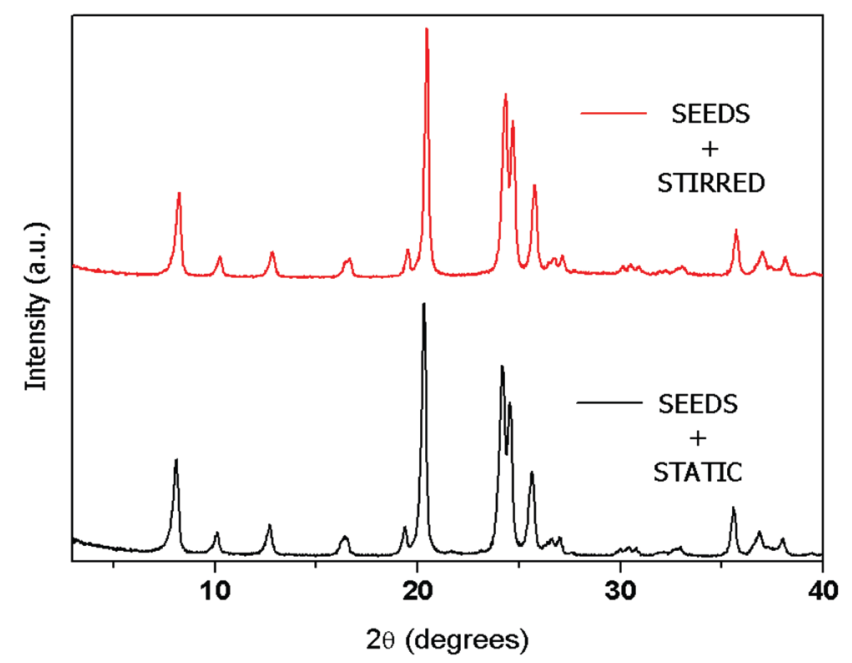

Figure 3. XRD of the ZSM-22 samples synthesized with seeds for static and agitated systems. 
The experiment with seeds and agitation showed the highest degree of crystallinity (100\% crystallinity of all the samples synthesized). Unlike the previous experiments without seeds, there was formation ZSM-22 in the system without agitation, but its percentage of crystallinity was slightly lower $(\sim 5 \%)$ compared to the agitated system.

In order to better understand the crystallization in static and agitated systems with addition of seeds, crystallization curves were constructed by periodic removal of samples, as shown in Figure 4.

In Figure 4, it is observed a rapid increase in the percentage of crystallinity of the samples from agitated systems - curves (a) and (c). It is noted that for the stirred seedless system the highest percentage of crystallinity was reached at $13 \mathrm{~h}$. As for the stirred system with seeds, $100 \%$ of crystallinity was reached at $7 \mathrm{~h}$. Therefore, the addition of seeds reduces by 4 hours the crystallization time to produce ZSM-22. In the case of static systems the addition of seed enables the formation of ZSM-22. However, the crystallization rate is slower than the one for stirred systems; the material is formed only after about $18 \mathrm{~h}$. This slower rate of crystallization is likely to be related to the difficulty in the diffusion of reactants within the reaction mixture to the surface of the growing crystal.

The average diameter of the ZSM-22 crystals was determined from the XRD patterns using the Scherrer equation, as indicated in Table 1. The data in the table indicates that crystals from seedless systems have smaller average diameters than the crystals obtained with seeds, as expected. This behavior may be due to two factors: 1) high speed stirring (400 rpm) prevents the formation of large crystals and 2) the use of seed introduces nuclei which speed the crystallization process resulting in larger crystals than for seedless systems. The calculated values for the average diameter of the crystals vary around $10 \%$, because the calculation uses the peak areas from the XRD profiles where errors may surge from axial deviations, heterogeneity of the particles, preferential orientation, among others [12] [13].

The ATG/DTG curves of different samples of ZSM-22 synthesized in this work were very similar (Figure 5), having as the main feature four distinct events of mass loss.

These events were attributed to the following processes: 1) dehydration of the zeolite; 2) loss of organic template weakly bound to the surface of the material; 3) thermal decomposition of the organic template strongly bound to the surface; 4) loss of structural water by the condensation of silanol groups [4] [14] [15] and elimination of the fragments formed from the template decomposition.

The measurement of weight loss at each step was performed using a DTG curve and the data is summarized in Table 2. The mass loss events related to the removal of organic template (II and III) are responsible for about

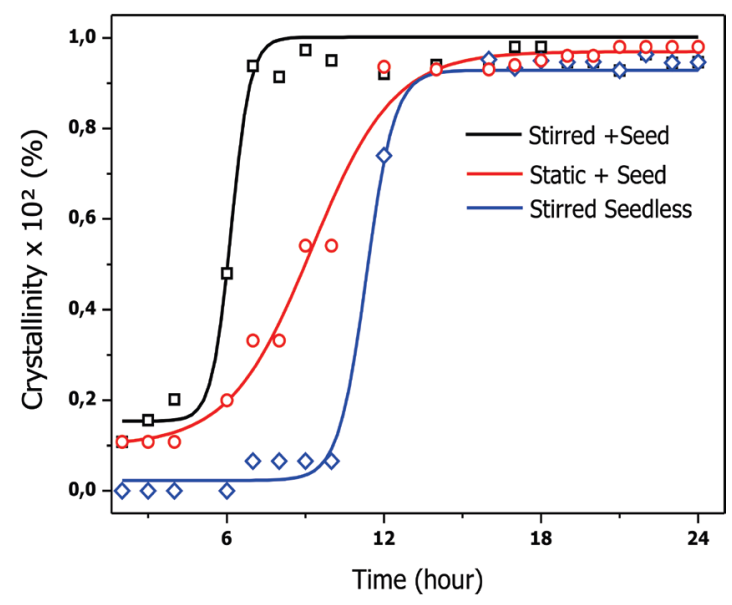

Figure 4. Crystallization curves. (a) Stirred + Seeds; (b) Static + Seeds; (c) Stirred Seedless.

Table 1. Average diameter of crystals obtained from the Scherrer equation for ZSM-22 samples with 24 hours of crystallization.

\begin{tabular}{ccccc}
\hline Sample & Colloidal Silica & Pyrolytic Silica & Stirred + Seeds & Static + Seeds \\
\hline Average Diameter of Crystals $(\mathrm{nm})$ & 32 & 28 & 40 & 39 \\
\hline
\end{tabular}




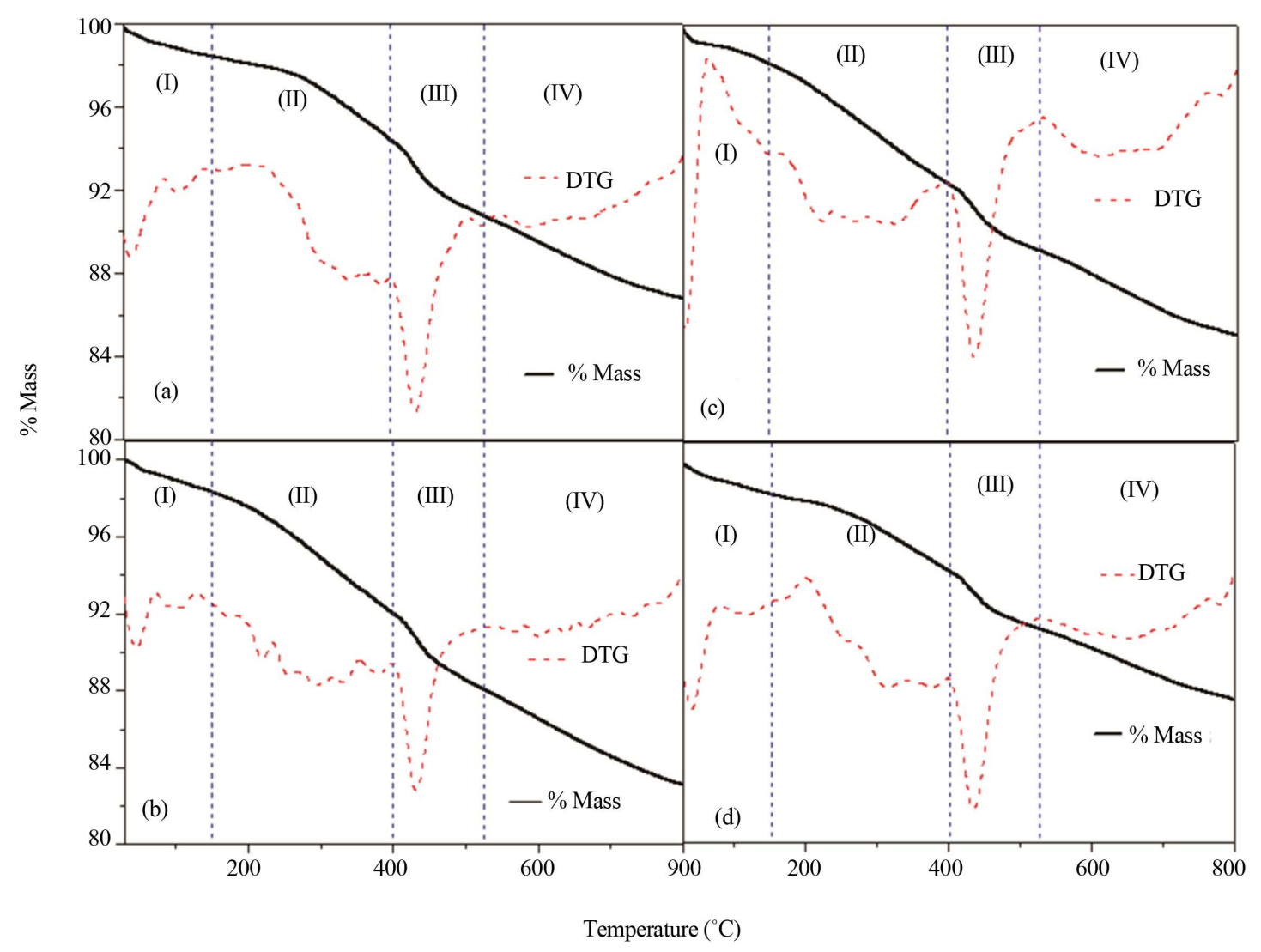

Figure 5. ATG/DTG curves. (a) Colloidal silica; (b) Pyrolytic silica; (c) Seed + agitation; d) Seeds + static.

Table 2. Temperature ranges and mass losses obtained by ATG.

\begin{tabular}{ccccccc}
\hline \multicolumn{1}{c}{ Sample } & \multicolumn{5}{c}{ Mass Loss (\%)/Temperature $\left({ }^{\circ} \mathbf{C}\right)$} \\
\hline Events & (I) $\mathbf{3 0}$ - 150 & (II) $\mathbf{1 5 0}-\mathbf{4 0 0}$ & (III) $\mathbf{4 0 0}$ - $\mathbf{5 2 5}$ & (IV) $\mathbf{5 2 5} \mathbf{- 8 0 0}$ & Total Loss (\%) \\
\hline Colloidal sílica & 1.36 & 4.01 & 3.57 & 3.96 & 13.98 \\
Pyrolytic silica & 1.66 & 6.32 & 3.94 & 4.94 & 16.87 \\
Seeds + stirred & 1.63 & 5.81 & 3.17 & 4.06 & 14.67 \\
Seeds + static & 1.59 & 4.02 & 3.04 & 3.71 & 12.35 \\
\hline
\end{tabular}

$2 / 3$ of the total sample mass loss.

Analysis of infrared spectroscopy for different ZSM-22 samples synthesized in this work are very similar. The spectrum for the most crystalline sample is shown in Figure 6. This curve shows five strong absorption bands in the region between 400 and $1500 \mathrm{~cm}^{-1}$. The bands in at 1090 and $470 \mathrm{~cm}^{-1}$ correspond to the asymmetric stretching vibration type and flexion (T-O), for the internal tetrahedra. The band near $790 \mathrm{~cm}^{-1}$ is due to symmetrical stretching of the external tetrahedra [16]. In the range between 1500 and $4000 \mathrm{~cm}^{-1}$ (not shown) there are only two bands corresponding to large surface hydroxyl groups and intracrystalline water molecules. A striking feature of the spectrum is a shoulder band near $1220 \mathrm{~cm}^{-1}$ [17], which is typical of ordered silicates.

\section{Conclusion}

This work investigated the crystallization of ZSM-22 in agitated and static systems, and with or without the addition of seeds. It was found that it is possible to synthesize ZSM-22 zeolite free of impurities at $160^{\circ} \mathrm{C}$ in the presence of 1,6-diaminohexane as the organic template using colloidal silica or pyrolytic silica as the silicon 


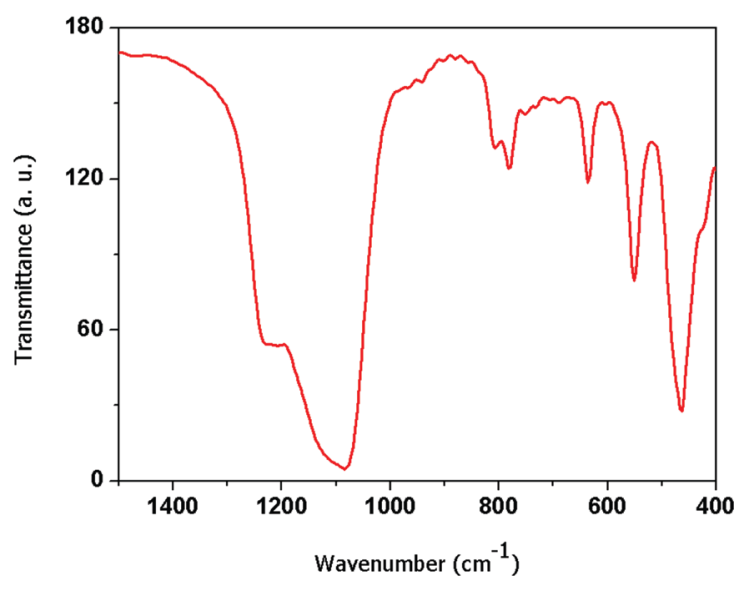

Figure 6. Infrared spectroscopy of ZSM-22 after 24 hours of crystallization without the addition of seeds with stirring.

source only if the system is stirred during crystallization. The shortest crystallization time with the highest crystallinity for agitated systems without seed material is $13 \mathrm{~h}$. The syntheses with silica gel or cogel (humid solid formed by amorphous aluminosilicate) even with agitation, do not result in ZSM-22. The use of silica gel formed ZSM-5, while the use of cogel resulted in an amorphous compound. The addition of seed material significantly accelerates the crystallization of ZSM-22, reducing the crystallization time to only $7 \mathrm{~h}$, for agitated systems using colloidal silica. Furthermore, the use of seeds allows the formation of ZSM-22 with colloidal silica without the necessity of agitation during crystallization. The average diameter data indicates that agitation at $400 \mathrm{rpm}$ during crystallization or using seeds (in the case of static systems) favor the rapid crystallization of ZSM-22 resulting in very small crystals in the range $28-40 \mathrm{~nm}$. Thermogravimetric analyses of the ZSM-22 samples show four events of mass loss and a total loss in the range between $12 \%$ to $14 \%$.

\section{Acknowledgements}

The authors thank the Coordination of Improvement of Higher Education Personnel (CAPES), the Financier of Studies and Projects (FINEP), PETROBRAS (SINFER2 Project) and the National Council for Scientific and Technological Development (CNPq) and the Federal University of Alagoas (UFAL) for financial support and facilities to conduct this study.

\section{References}

[1] Ernst, S., Weitkamp, J., Martens, J.A. and Jacobs, P.A. (1989) Synthesis and Shape-Selective Properties of ZSM-22. Applied Catalysis, 48, 137.

[2] Kumar, N., Lindfors, L.E. and Byggningsbacka, R. (1996) Synthesis and Characterization of H-ZSM-22, Zn-H-ZSM-22 and Ga-H-ZSM-22 Zeolite Catalysts and Their Catalytic Activity in the Aromatization of n-Butane. Applied Catalysis, 139, 189.

[3] Kumar, R. and Ratnasamy, P. (1989) Somerization and Formation of Xylenes over ZSM-22 and ZSM-23 Zeolites. Journal of Catalysis, 116, 440.

[4] Borade, R.B., Adnot, A. and Kaliaguine, S. (1991) Acid Sites in AI-ZSM-22 and Fe-ZSM-22. Zeolite, 11, 710.

[5] Cundy, C.S. and Cox, P.A. (2005) The Hydrothermal Synthesis of Zeolites: Precursors, Intermediates and Reaction Mechanism. Microporous and Mesoporous Materials, 82, 78.

[6] Thompson, R.W. and Robson, K.P.H. (2001) Nucleation, Growth, and Seeding in Zeolite Synthesis. In: Robson, H., Ed., Verifield Syntheses of Zeolitic Materials, Elsevier, Amsterdam, 21-23.

[7] Verboekend, D., Chabaneix, A.M., Thomas, K., Gilson, J.P. and Ramírez, J.P. (2011) Mesoporous ZSM-22 Zeolite Obtained by Desilication: Peculiarities Associated with Crystal Morphology and Aluminium Distribution. Cryst. Eng. Comm., 13, 3408-3416.

[8] Schmidt, W., Toktarev, A.V., Schuth, F., Lone, K.G. and Unger, K. (2001) Studies in Surface Science and Catalysis, 
135-190.

[9] Klug, H.P. and Alexander, L.E. (1954) X-Ray Diffraction Procedures. John Wiley \& Sons, New York.

[10] Treacy, M.M.J. and Higgins, J.B. (2007) Collection of Simulated XRD Powder Diffraction Patterns for Zeolites. 5th Edition, Elsevier, Amsterdam.

[11] Derewinski, M. and Machowska, M. (2004) Effect of Stirring on the Selective Synthesis of MEL or TON Zeolites in the Presence of 1,8-Diaminooctane. In: van Steen, E., Callanan, L.H., \& Claeys, M., Eds., Studies in Surface Science and Catalysis, Elsevier, Amsterdam, 349-354.

[12] Connolly, J.R. (2013) Introduction to X-Ray Powder Diffraction. http://epswww.unm.edu/xrd/xrdclass/01-XRD-Intro.pdf

[13] Connolly, J.R. (2013) Introduction Quantitative X-Ray Diffraction Methods. http://epswww.unm.edu/xrd/xrdclass/09-Quant-intro.pdf

[14] Franklin, K.R. and Lowe, B.M. (1988) Hydrothermal Crystallization of (Hexane-1,6-Diamine)-Silicalite-1. Zeolite, 8, 495.

[15] Singh, A.P. and Reddy, K.R. (1994) Synthesis, Characterization, and Catalytic Activity of Gallosilicate Analogs of Zeolite ZSM-22. Zeolite, 14, 290.

[16] Silva, A.O.S. (2004) Sintese e Caracterização de Catalisadores de Ferro e Cobalto Suportados nas Zeólitas HZSM-12 e HZSM-5 para a Conversão de Gás de Síntese em Hidrocarbonetos. Universidade Federal do Rio Grande do Norte, Natal.

[17] Simon, M.W., Suib, S.T. and Young, C.O. (1994) Synthesis an Characterization of ZSM-22 Zeolites and Their Catalytic Behavior in 1-Butene Isomerization Reactions. Journal of Catalysis, 147, 484. 